\title{
Efficacy of suction blister epidermal grafting with concomitant phototherapy in vitiligo treatment
}

\author{
Tomasz Iwanowski ${ }^{1}$, Piotr Szlązak ${ }^{1}$, Alicja Rustowska², Małgorzata Sokołowska-Wojdyło³ \\ ${ }^{1}$ Dermedica, Gdansk, Poland \\ ${ }^{2}$ Medpharma, Warsaw, Poland \\ ${ }^{3}$ Department of Dermatology, Venerology and Allergology, Medical University of Gdansk, Gdansk, Poland
}

Adv Dermatol Allergol 2018; XXXV (6): 592-598

DOI: https://doi.org/10.5114/pdia.2017.71257

\begin{abstract}
Introduction: There are many surgical methods for vitiligo treatment that have been used for over 30 years. Suction blister epidermal grafting (SBEG) is considered one of the simplest and most effective of them.

Aim: To determine how effective suction blister grafts with concomitant phototherapy are in vitiligo treatment. Material and methods: The study was conducted on 10 patients with vitiligo that was resistant to previous treatment including phototherapy in monotherapy. Involvement of affected sites was different for every patient. We used cryotherapy for blistering at the recipient site and an automatic suction device for blistering at the donor site. The blister was separated from the donor site and fixed with dressing to the recipient site. After removing the final dressing (about 7 days after SBEG) patients started phototherapy ( 6 patients had UVB $311 \mathrm{~nm}$ and 4 had PUVA). All patients treated with UVB $311 \mathrm{~nm}$ were qualified for treatment in our clinic and the method was chosen according to expert recommendations from the European Dermatology Forum (EDF) Guidelines for Vitiligo where narrowband (NB) UVB is the phototherapy of choice. Three patients who had PUVA therapy were treated with this method in other clinical centers and sent to us only to undergo SBEG. One patient had previously received UVB $311 \mathrm{~nm}$ for 3 months, which showed no effects. Repigmentation of lesions was evaluated at 3 and 6 months after the surgical procedure.

Results: Ten patients ( 9 females with a mean age of 36.88 years and 1 man aged 39 years) were enrolled in the study. Nine patients showed progressive repigmentation at 3 and 6 months follow-up with a rate varying from 13 to $76 \%$ (mean: $44.5 \%$ ) and 35 to $100 \%$ (mean: 67.5\%). One patient showed 5\% depigmentation at a visit after 6 months in comparison to the follow-up visit 3 months after SBEG.

Conclusions: With this technique, patients who did not respond to the usual treatments showed very good repigmentation over a 6-month follow-up. There were no side effects such as scarring.
\end{abstract}

Key words: suction blister, PUVA, UVB $311 \mathrm{~nm}$, repigmentation, vitiligo, planimetry.

\section{Introduction}

Vitiligo is an acquired skin disorder of great cosmetic significance. It affects approximately $0.1-2 \%$ of the world population, varying in different world regions [1, 2]. Topical corticosteroids or calcineurin inhibitors are the most valuable treatments for localized vitiligo [3]. Phototherapy is currently the most widely used for large vitiligous areas and smaller lesions resistant to other therapies [4-8]. Narrow-band UVB is considered by many to be equally effective to psoralen and ultraviolet $A$ (PUVA) and thus is used as the first choice therapy for most patients $[3,9,10]$. In spite of this multiplicity of affordable treatment options, many physicians are still frustrated with results and prescribe no treatment at all. Because of these disappointing outcomes, several surgical methods were introduced in the hope of achieving a more encouraging response. Surgical techniques are reserved for patients with stable vitiligo that is unresponsive to conventional therapies [11-14]. A number of dermatosurgery techniques are available to promote repigmentation of vitiligo: mini- or punch grafts, split-thickness skin grafts, cultured epidermal sheets, cultured melanocyte suspensions, follicular grafts and suction blister epidermal grafts [15-26]. Each procedure has its advantages

Address for correspondence: Tomasz Iwanowski, Dermedica, 24 Batalionów Chłopskich St, 81-415 Gdynia, Poland, phone: +48 501926 907, e-mail: tomasziwan@gmail.com Received: 17.07.2017, accepted: 9.10.2017. 
and disadvantages. Minipunch grafting, though a simple outpatient procedure, is not preferred over cosmetically important sites because of the frequently associated cobblestoning $[27,28]$. Split thickness grafting has the advantage that it is a simple procedure by which large areas of vitiligo can be covered in a single sitting and no special equipment is required. However, scar formation at the donor site, milia, partial loss of grafts, and thick margins of the grafts are commonly associated side effects [27]. The suction blister technique has the shortcoming that it is time consuming and only small areas can be covered in a single sitting $[14,27]$. However, it is ideal for covering small, cosmetically important sites. Among these methods, the highest success rates have been achieved with split-thickness skin grafts and epidermal blister grafts. However, we consider SBEG to be less painful for the patient, easier and practically free from side effects such as scarring. Suction blisters were first introduced by Kiistala and Mustakallio in 1964 by using an angiosterrometer [29]. Its use in replacing achromic skin was first reported by Falabella in 1971 [30]. Application of epithelial grafts nowadays is a common treatment technique for vitiligo. Unfortunately failures are still anticipated in many cases. For better results, phototherapy or photochemotherapy of donor and recipient sites can also be performed before and after grafting [11, 14, 27]. The purpose of this work is to evaluate how effective suction blister grafts with concomitant phototherapy are in treatment of resistant vitiligo cases that had failed to respond to classic phototherapy. Many studies have been performed to investigate the efficacy of vitiligo treatment modalities and a variety of assessment methods have been used to evaluate the response to therapy. Many of them rely on the subjective assessment of repigmentation by the investigator. There is still no consensus that would allow an objective and reliable evaluation of treatment results. We decided to use planimetric measurement of vitiligous areas with a tool that is free to use - Image (https:// imagej.nih.gov/ij/index.html).

\section{Aim}

The aim of this study was to determine how effective suction blister grafts with concomitant phototherapy are in vitiligo treatment.

\section{Material and methods}

Ten patients (9 females with a mean age of 36.88 years and 1 man aged 39 years) were enrolled in the study (Table 1). All had stable localized vitiligo and they did not respond satisfactorily to classic phototherapy treatment although they had received this for at least 3 months. Patients under 18 years old, cases with active disease (with new crops of depigmented skin in the last 3 months), and Koebner-positive cases were excluded from the study. Full history taking, general and local examination, and photography of the lesion(s) were done for each patient. Informed written consent was obtained in all cases. The day before surgery cryotherapy was performed at the vitiligo-affected recipient site. Cryotherapy was performed with nitrous suboxide through one cycle of 10-15 s. On the day of surgery, a donor site was selected on the brachial area (with normal skin) and the area was cleaned with Kodan forte solution. The site was attached to the transparent blister-forming device and vacuum extractor apparatus to produce a high negative pressure ( -0.4 bar; 6 psi) (Figure $1 \mathrm{~A})$. After about $2 \mathrm{~h}$ of application of suction, every blister was ready (Figure $1 \mathrm{~B}$ ). Then all bullae in the recipient site were converted into erosions by removing the roofs of the bullae with scissors. Every erosion was round and had a diameter of approximately $5-7 \mathrm{~mm}$. After that suction blisters at the donor site were cut off one by one with scissors along

Table 1. List of all patients

\begin{tabular}{|c|c|c|c|c|}
\hline Patient number & Gender & Age & $\begin{array}{l}\text { Vitiligous area subjected } \\
\text { to SBEG }\end{array}$ & Phototherapy \\
\hline 1 & Male & 39 & Hand - 1 area & UVB 311 nm \\
\hline 2 & Female & 41 & Elbow - 2 areas & UVB 311 nm \\
\hline 3 & Female & 42 & Neck - 1 area & UVB 311 nm \\
\hline 4 & Female & 48 & Face, neck - 2 areas & UVB 311 nm \\
\hline 5 & Female & 37 & Calf - 1 area & UVB 311 nm \\
\hline 6 & Female & 29 & Back - 1 area & UVB 311 nm \\
\hline 7 & Female & 31 & Hand -3 areas & PUVA \\
\hline 8 & Female & 41 & Back - 1 area & PUVA \\
\hline 9 & Female & 23 & Foot -1 area & PUVA \\
\hline 10 & Female & 40 & Hand -1 area & PUVA \\
\hline
\end{tabular}



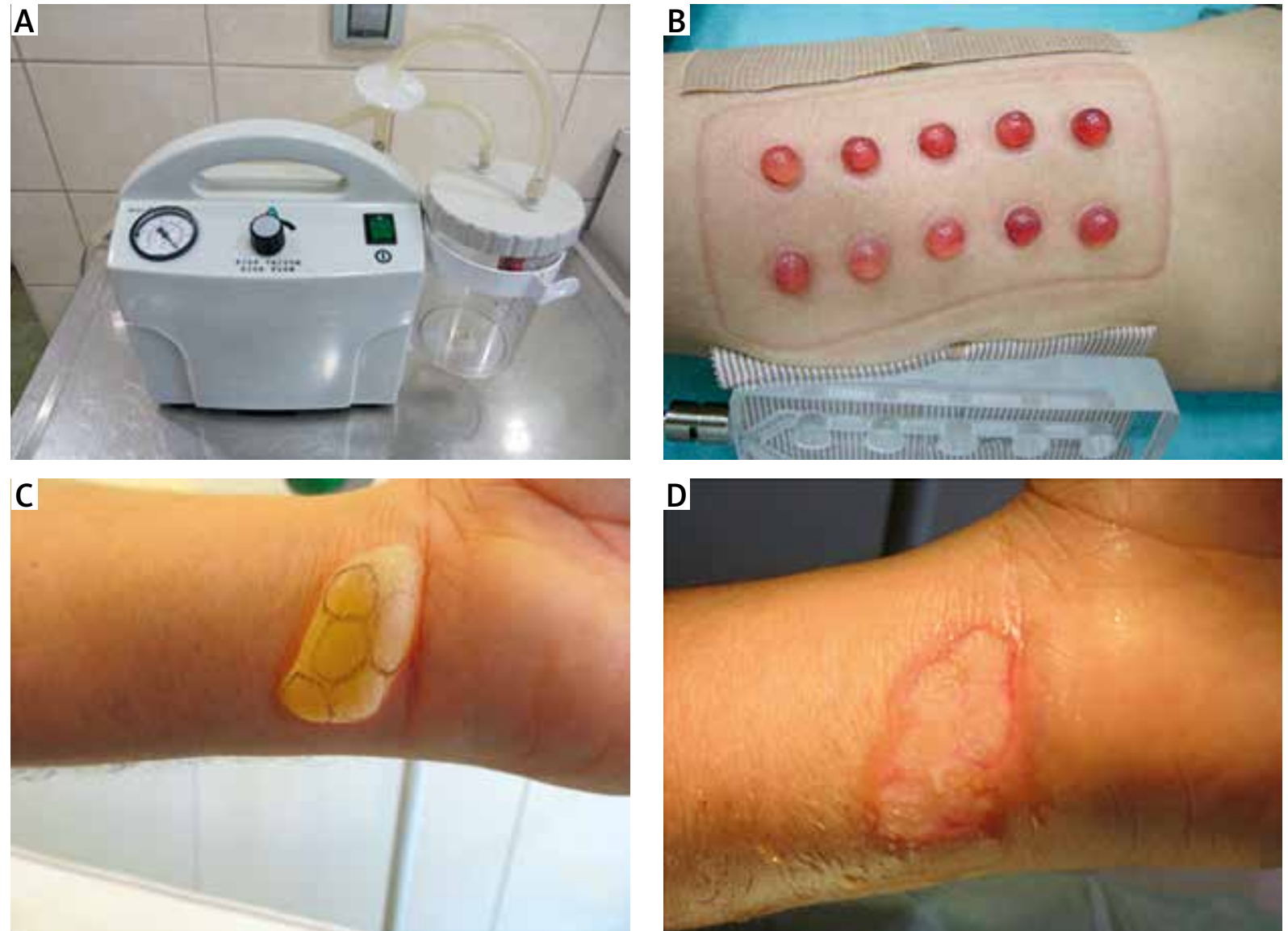

Figure 1. A - Vacuum extractor apparatus, B - suction blisters in the donor site and transparent blister forming device, $\mathbf{C}$ - bullae in the recipient site, $\mathbf{D}$ - suction blisters placed on the recipient site

the blister base, placed on object glass and then finally placed on the recipient site (Figures 1 C, D). Forceps and a surgical blade were used to extend the graft to its largest size so that it could cover whole erosion at the vitiliginous area. The harvested layer consisted of only epithelium with no dermal components. The donor and the recipient sites were dressed with Bactigras and gauze, which was bound in place with an adhesive plaster. The dressing was changed every day and removed completely after a week. Phototherapy was initiated immediately after removing the final dressing. UVB $311 \mathrm{~nm}$ was chosen as the phototherapy of choice for 6 of our patients according to expert recommendations from the EDF Guidelines. PUVA therapy was continued in 3 of 4 patients sent from other clinical centers to undergo SBEG. In one patient we changed UVB $311 \mathrm{~nm}$ to PUVA because of therapeutic failure after 3 months of phototherapy. All patients were followed postoperatively for 6 months. Finally, repigmentation rates were evaluated by comparing images of the lesions at 3 months and 6 months after surgery.

Still there is no consensus about the choice of evaluation methods and outcome parameters used in surgical vitiligo studies. These results are thus non- comparable data. Most methods are based on visual assessment, which may lead to a highly subjective outcome. We found only three studies that mention the use of a more objective measurement tool. The planimetric measurement used by Boersma et al. [31] was in our opinion the most accurate, and we used the same method in our study.

\section{Results}

In this study, 10 patients (9 female and 1 male) with stable vitiligo were evaluated for response following suction blister grafting, with pre- and post-graft phototherapy. The age of our male patient was 39 and female patients' medium age was 36.88. Responses to treatment at different follow-up evaluations are presented in Table 2. Nine patients showed progressive repigmentation at 3 and 6 months follow-up with a rate varying from 13 to $76 \%$ (mean: $44.5 \%$ ) and 35\% to $100 \%$ (mean: $67.5 \%$ ) (Figures 2 A, B). Repigmentation rates between the first and second follow-up visit varied from $14 \%$ to $100 \%$. One patient showed 5\% depigmentation at a visit after 6 months in comparison to the follow-up visit 3 months after SBEG. One patient presented the Koebner phenom- 
Table 2. Response to treatment after 3 and 6 months

\begin{tabular}{|c|c|c|c|c|c|c|}
\hline $\begin{array}{l}\text { Patient } \\
\text { number }\end{array}$ & $\begin{array}{l}\text { Recipient } \\
\text { vitiligous area } \\
\text { surface before } \\
\text { SBEG (A) }\left[\mathrm{mm}^{2}\right]\end{array}$ & $\begin{array}{l}\text { Recipient area } \\
\text { vitiligous surface } \\
3 \text { months after } \\
\text { SBEG (B) }\left[\mathrm{mm}^{2}\right]\end{array}$ & $\begin{array}{l}\text { Recipient area } \\
\text { vitiligous surface } \\
6 \text { months after } \\
\text { SBEG (C) }\left[\mathrm{mm}^{2}\right]\end{array}$ & $\begin{array}{l}\text { A-B }\left[\mathrm{mm}^{2}\right] \\
\text { (and \% } \\
\text { of repigmentation } \\
\text { area) }\end{array}$ & $\begin{array}{l}\mathrm{A}-\mathrm{C}\left[\mathrm{mm}^{2}\right] \\
\text { (and \% } \\
\text { of repigmentation } \\
\text { area) }\end{array}$ & $\begin{array}{c}\mathrm{B}-\mathrm{C}\left[\mathrm{mm}^{2}\right] \\
\text { (and \% } \\
\text { of repigmentation } \\
\text { area) }\end{array}$ \\
\hline 1 & 540 & 207 & 105 & $333(52)$ & $435(81)$ & $102(51)$ \\
\hline 2.1 & 262 & 169 & 60 & $93(36)$ & $202(77)$ & $109(65)$ \\
\hline 2.2 & 165 & 143 & 103 & $22(13)$ & $62(38)$ & $40(28)$ \\
\hline 3 & 405 & 293 & 0 & $112(28)$ & 405 (100) & $293(100)$ \\
\hline 4.1 & 764 & 270 & 40 & $494(65)$ & 724 (95) & $230(85)$ \\
\hline 4.2 & 4642 & 1258 & 183 & $3384(73)$ & 4459 (96) & $1075(85)$ \\
\hline 5 & 941 & 226 & 118 & 715 (76) & $823(88)$ & $108(48)$ \\
\hline 6 & 820 & 432 & 152 & $388(48)$ & $668(82)$ & $280(65)$ \\
\hline 7.1 & 253 & 149 & 59 & $104(41)$ & $194(77)$ & $90(60)$ \\
\hline 7.2 & 384 & 212 & 164 & $172(45)$ & $220(57)$ & $48(23)$ \\
\hline 7.3 & 144 & 81 & 70 & $63(44)$ & $74(51)$ & $11(14)$ \\
\hline 8 & 1695 & 573 & 215 & $1122(67)$ & $1480(88)$ & $358(63)$ \\
\hline 9 & 2685 & 749 & 265 & $1936(72)$ & $2420(90)$ & $484(65)$ \\
\hline 10 & 209 & 126 & 133 & $83(40)$ & $76(35)$ & $-7(-5)$ \\
\hline
\end{tabular}
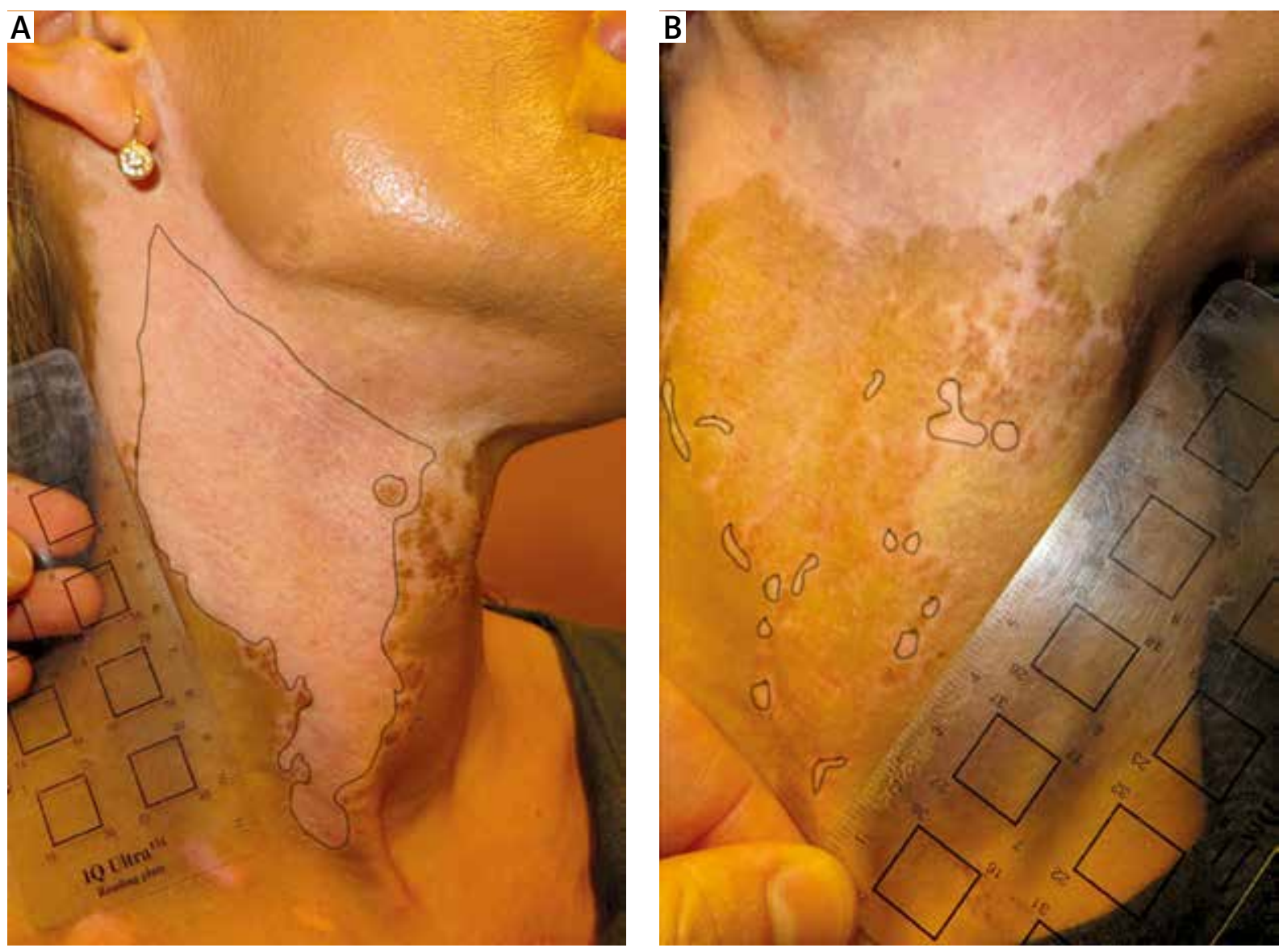

Figure 2. A - Patient 4, area 2 (neck) - before SBEG, B - patient 4, area 2 (neck) - 6 months after SBEG (after transferring 40 suction blisters) 
enon in the donor area but vitiligous patches repigmented 2 months after starting phototherapy. We observed a significantly better repigmentation rate in patients with concomitant UVB $311 \mathrm{~nm}$ therapy than PUVA, which was $82.125 \%$ after 6 months and $69.4 \%$ after 6 months, respectively.

\section{Discussion}

Currently, several medical treatments for vitiligo are available, including topical and systemic corticosteroid, photochemotherapy using PUVA, narrow-band UVB, human placental extracts, antioxidants, and low-energy laser irradiation [32, 33]. Despite the availability of these therapeutic options, many patients with vitiligo fail to achieve satisfactory results. For patients whose vitiligo has been nonresponsive to medical treatments and has been stable, surgical therapies are viable alternatives. Autologous skin grafts can be obtained from uninvolved skin using several dermatosurgery techniques [1]. Each method has its pros and cons. The mini-graft is simple and non-expensive, but it has the highest rate of adverse effects, with a $35 \%$ risk of cobblestone appearance at the recipient site and hypopigmentation and keloid formation at the donor site $[34,35]$. Thin split-thickness grafting has the highest mean success rate (87\%) according to a systematic review by Njoo et al. [27] Transplantation of melanocytes cultured in vitro from a small piece of donor skin is very expensive and requires special and advanced laboratory facilities that are currently available only at a few academic centers [9]. Suction blister grafting is accomplished by suction of pigmented epidermis from the dermis and its transfer to achromic areas. When the epidermal grafts remain in contact with the denuded surface of the recipient vitiliginous area for about a week, the melanocytes readily migrate to the graft bed, resulting in repigmentation. Therefore, this procedure is actually "melanocyte transfer" and the epidermis obtained through suction blisters is only a carrier [36, 37]. In most studies in the literature, 1 week after the surgical procedure phototherapy was used to induce proliferation of melanocytes at the recipient sites [38-40]. However, in two studies the authors proved that it is not necessary for obtaining repigmentation in vitiligous areas [41, 42]. The repigmentation rate in all these studies, according to the review by Njoo et al., was $87 \%$, whereas Ozdemir et al. reported rates of $25-65 \%[27,43]$. In a study simi- lar to ours, Maleki et al. evaluated 10 patients with refractory vitiligo who were treated with a suction blister graft and subsequent PUVA therapy and reported over 90\% repigmentation in 7 patients [44]. In our study, blister grafting with concomitant phototherapy showed repigmentation over $90 \%$ at the 6 months follow-up in 3 patients and over $70 \%$ in 6 patients, which according to the most popular criteria for the repigmentation rate (> 90\% = complete, $71-90 \%=$ good) is a very good result. Although phototherapy alone failed to help the same patients with the same dosage, it succeeded here in restoration of pigmentation in a short time (after 3 months mean $44.5 \%$ and after 6 months mean 67.5\%). We observed the Koebner phenomenon in the donor area of 1 patient with stable vitiligo like a few authors before $[45,46]$, but like Kim and Kang [47] we believe that it is not problematic. We compared in detail our results to another study conducted by Maleki et al. [42] in which no concomitant phototherapy was performed after SBEG. He evaluated 10 lesions in 10 patients and graded repigmentation rates as 'complete' (> 90\%), 'good' (71-90\%), 'moderate' (51-70\%) and 'poor' (<50\%). We evaluated 10 patients with 14 vitiligous areas and for this comparison graded same repigmentation rates. The results are shown in Table 3. Interestingly, repigmentation rates in our patients are worse than the group of Maleki et al. that was not subjected to phototherapy after the surgical procedure. It may be the result of ethnic differences, but to confirm that another study on our patients should be performed where the control group does not have concomitant phototherapy.

\section{Conclusions}

The advantages of this technique include low cost, absence of scarring at donor or recipient sites and the possibility of reusing the donor site. Furthermore, it does not have the limitations of other tissue grafts (minigrafts, pinch grafts, or thin or ultrathin split thickness grafts), which often lead to mismatch in texture and color, not acceptable at cosmetically prominent sites. The disadvantages are that it is time-consuming and not suitable for large areas, which have to be operated on a few times to achieve repigmentation. In summary, the results of our study indicate that SBEG combined with phototherapy can provide rapid, safe, effective treatment for small, stable vitiligo.

Table 3. Comparison of repigmentation rate in Maleki et al. study vs. our patients

\begin{tabular}{|c|c|c|c|c|c|c|c|c|}
\hline \multirow{3}{*}{$\begin{array}{l}\text { Follow-up } \\
\text { visit } \\
\text { [months] }\end{array}$} & \multicolumn{8}{|c|}{ Response to treatment } \\
\hline & \multicolumn{2}{|c|}{ Mild } & \multicolumn{2}{|c|}{ Moderate } & \multicolumn{2}{|c|}{ Good } & \multicolumn{2}{|c|}{ Complete } \\
\hline & Maleki et al. & Our study & Maleki et al. & Our study & Maleki et al. & Our study & Maleki et al. & Our study \\
\hline 3 & $0 \%$ & $57 \%$ & $20 \%$ & $21 \%$ & $20 \%$ & $22 \%$ & $60 \%$ & $0 \%$ \\
\hline 6 & $0 \%$ & $36 \%$ & $20 \%$ & $43 \%$ & $10 \%$ & $14 \%$ & $70 \%$ & $7 \%$ \\
\hline
\end{tabular}




\section{Conflict of interest}

The authors declare no conflict of interest.

\section{References}

1. Scherschun L, Kim JJ, Lim HW. Narrow-band ultraviolet B is a useful and well-tolerated treatment for vitiligo. J Am Acad Dermatol 2001; 44: 999-1003.

2. Majumder MP. Genetic and prevalence of vitiligo vulgaris. In: Vitiligo. Hann BK, Nordlund JJ (eds). Blackwell Sience, Hoboken, New Jersey 2000; 18-20.

3. Ortonne JP. Pigmentary disorders. In: Dermatology. Bolognia JL, Jorrizo JL, Rapini RP (eds). $2^{\text {nd }}$ ed. Mosby Elsevier, Philadelphia 2008; 913-20.

4. Löntz W, Olsson MJ, Moellmann G, Lerner AB. Pigment cell transplantation for treatment of vitiligo: a progress report. J Am Acad Dermatol 1994; 30: 591-7.

5. Sharque KE. Vitiligo. Clin Exp Dermatol 1984; 9: 117-26.

6. Kenney JA Jr. Vitiligo. Dermatol Clin 1988; 6: 425-34.

7. Jimbow K. Vitiligo: therapeutic advances. Dermatol Clin 1998; 16: 399-407.

8. Grimes PE. Vitiligo: an overview of therapeutic approaches. Dermatol Clin 1993; 11: 325-38.

9. Anstey AV. Disorders of skin colour. In: Rook's Textbook of Dermatology. Burns T, Breathnach S, Cox N, Griffiths CEM (eds). Vol. 58. Wiley Blackwell, Hoboken, New Jersey 2010; 47-50.

10. Westerhof W, Nieuweboer-Krobotova L. Treatment of vitiligo with UV-B radiation vs topical psoralen plus UV-A. Arch Dermatol 1997; 133: 1525-8.

11. Suga Y, Butt KI, Takimoto R, et al. Successful treatment of vitiligo with PUVA pigmented autologous epidermal grafting. Int J Dermatol 1996; 35: 518-22.

12. Falabella R, Escobar C, Borrero I. Treatment of refractory and stable vitiligo by transplantation of in vitro cultured epidermal autografts bearing melanocytes. J Am Acad Dermatol 1992; 26: 230-6.

13. Falabella R, Arrunategni A, Barona MI, Alzate A. The minigrafting test for vitiligo: detection of stable lesions for melanocytes transplantation. J Am Acad Dermatol 1995; 32: 228-32.

14. Hann SK, Bong HW, Park Y. Treatment of stable vitiligo with autologous epidermal grafting and PUVA. J Am Acad Dermatol 1995; 32: 943-8.

15. Falabella R, Barona MI. Update on skin repigmentation therapies in vitiligo. Pigment Cell Melanoma Res 2009; 22: 42-65.

16. Agrawal K, Agrawal A. Vitiligo: repigmentation with dermabrasion and thin split thickness skin graft. Dermatol Surg 1995; 21: 295-300.

17. Falabella R. Repigmentation of segmental vitiligo by autologous minigrafting. J Am Acad Dermatol 1983; 9: 514-21.

18. Khunger N, Kathuria SD, Ramesh V. Tissue grafts in vitiligo surgery - past, present, and future. Indian I Dermatol 2009; 54: 150-8.

19. Lahiri K. Evolution and evaluation of autologous mini punch grafting in vitiligo. Indian J Dermatol 2009; 54: 159-67.

20. Lahiri K, Malakar S, Sarma N, Banerjee U. Repigmentation of vitiligo with punch grafting and narrow-band UV-B (311 nm) a prospective study. Int I Dermatol 2005; 45: 649-55.

21. Gauthier Y, Surleve-Bazeille JE. Autologous grafting with noncultured melanocytes: a simplified method for treatment of depigmented lesions. J Am Acad Dermatol 1992; 26: 191-4.
22. Guerra L, Capurro S, Melchi F, et al. Treatment of 'stable' vitiligo by Timedsurgery and transplantation of cultured epidermal autografts. Arch Dermatol 2000; 136: 1380-9.

23. Kim CY, Yoon TJ, Kim TH. Epidermal grafting after chemical epilation in the treatment of vitiligo. Dermatol Surg 2001; 27: 855-6.

24. Falabella R. Grafting and transplantation of melanocytes for repigmenting vitiligo and other types of leukoderma. Int J Dermatol 1989; 28: 363-9.

25. Koga M. Epidermal grafting using the tops of suction blisters in the treatment of vitiligo. Arch Dermatol 1988; 124: 1656-8.

26. Hong W, Hu DN, Qian GP, et al. Treatment of vitiligo in children and adolescents by autologous cultured pure melanocytes transplantation with comparison of efficacy to results in adults. J Eur Acad Dermatol Venereol 2011; 25: 538-43.

27. Njoo MD, Westerhof W, Bos JD, et al. A systematic review of autologous transplantation methods in vitiligo. Arch Dermatol 1998; 134: 1543-9.

28. Gupta S, Jain VK, Saraswat PK, Gupta S. Suction blisters epidermal grafting versus punch skin grafting in recalcitrant and stable vitiligo. Dermatol Surg 1999; 25: 955-8.

29. Kiistala U, Mustakallio KK. In vivo separation of epidermis by production of suction blisters. Lancet 1964; 1: 1444-5.

30. Falabella R. Epidermal grafting: an original technique and its application in achromic and granulating areas. Arch Dermatol 1971; 104: 592-600.

31. Boersma B, Weterhof W, Bos J. Repigentation in vitiligo vulgares by autologous minigrafting: results in nineteen patients. J Am Acad Dermatol 1995; 33: 990-5.

32. Grimes PE. New insights and new therapies in vitiligo. JAMA 2005; 293: 730-5.

33. Shaffrali F, Gawkrodger D. Management of vitiligo. Clin Exp Dermatol 2000; 25: 575-9.

34. Babu A, Thappa DM, Jaisankar TJ. Punch grafting versus suction blister epidermal grafting in the treatment of stable lip vitiligo. Dermatol Surg 2008; 34: 166-78.

35. Rusfianti M, Wirohadidjodjo YW. Dermatosurgical techniques for repigmentation of vitiligo. Int J Dermatol 2006; 45: 411-7.

36. Horikawa T, Mishima Y, Nishino K, Ichihashi M. Horizontal and vertical pigment spread into surrounding piebald epidermis and hair follicles after suction blister epidermal grafting. Pigment Cell Res 1999; 12: 175-80.

37. Gupta S, Sandhu K, Kanwar A, Kumar B. Melanocyte transfer via epidermal grafts for vitiligo of labial mucosa. Dermatol Surg 2004; 30: 45-8.

38. Lee AY, Jang JH. Autologous epidermal grafting with PUVAirradiated donor skin for the treatment of vitiligo. Int J Dermatol 1998; 37: 551-4.

39. Ortonne JP, MacDonald DM, Micoud A, Thivolet J. PUVA-induced repigmentation of vitiligo: a histochemical (split-DOPA) and ultrastructural study. Br J Dermatol 1979; 101: 1-12.

40. Awad SS, Abdel-Raof H, El-Din WH, El-Domyati M. Epithelial grafting for vitiligo requires ultraviolet A phototherapy to increase success rate. J Cosmet Dermatol 2007; 6: 119-24.

41. Nanda S, Relhan V, Grover C, Reddy BS. Suction blister epidermal grafting for management of eyelid vitiligo. Dermatol Surg 2006; 32: 391-2.

42. Maleki M, Banihashemi M, Sanjari V. Efficacy of suction blister epidermal graft without phototherapy for locally stable and resistant vitiligo. Indian I Dermatol 2012; 57: 282-4.

43. Ozdemir M, Cetinkale O, Wolf R, et al. Comparison of two surgical approaches for treating vitiligo: a preliminary study. Int J Dermatol 2002; 41: 135-8. 
44. Maleki M, Javidi Z, Ebrahimirad M. Treatment of vitiligo with blister grafting technique. Iranian I Dermatol 2008; 11: 55-9.

45. Njoo MD, Das PK, Bos JD, Westerhof W. Association of the Kobner phenomenon with disease activity and therapeutic responsiveness in vitiligo vulgaris. Arch Dermatol 1999; 135: 407-13.

46. Gupta S, Kumar B. Epidermal grafting in vitiligo: influence of age, site of lesion, and type of disease on outcome. J Am Acad Dermatol 2003; 49: 99-104.

47. Kim HY, Kang KY. Epidermal grafts for treatment of stable and progressive vitiligo. J Am Acad Dermatol 1999; 40: 412-7. 